\title{
Generation of two-photon states with arbitrary degree of entanglement via nonlinear crystal superlattices
}

\author{
Alfred B. U'Ren ${ }^{1,3,4}$, Reinhard K. Erdmann ${ }^{2,4}$, Manuel de la Cruz-Gutierrez ${ }^{1,4}$, Ian A. Walmsley ${ }^{1}$ \\ ${ }^{1}$ Clarendon Laboratory, Oxford University, Parks Road, Oxford, OX1 3PU, England \\ ${ }^{2}$ Sensors Directorate, Air Force Research Laboratory, Rome, NY, USA \\ ${ }^{3}$ Centro de Investigación Científica y Educación Superior de Ensenada (CICESE), Baja California, 22860, Mexico \\ ${ }^{4}$ The Institute of Optics, University of Rochester, Rochester, New York 14627, USA
}

(Dated: February 5, 2020)

\begin{abstract}
We demonstrate a general method of engineering the joint quantum state of photon pairs produced in spontaneous parametric downconversion (PDC). The method makes use of a superlattice structure of nonlinear and linear materials, in conjunction with a broadband pump, to manipulate the group delays of the signal and idler photons relative to the pump pulse, and realizes photon pairs described by a joint spectral amplitude with arbitrary degree of entanglement. This method of group delay engineering has the potential of synthesizing a broad range of states including factorizable states crucial for quantum networking and states optimized for Hong-Ou-Mandel interferometry. Experimental results for the latter case are presented, illustrating the principles of this approach.
\end{abstract}

PACS numbers: 42.50.Ar, 03.67.Lx

Quantum interference between single-photon wavepackets lies at the heart of novel quantum-enhanced communication and computation technologies. Such interference depends crucially on the spatio-temporal mode structure of the photons used. Therefore it is important to develop techniques that enable the generation of quantum states with the specific modal character required for different applications. In particular, entangled photon pairs provide an important resource for several quantum information processing (QIP) protocols, and single photons in pure states a resource for others. While great progress has been made in the generation of single photons on-demand[1], the process of parametric downconversion (PDC) constitutes a practical room-temperature source of non-classical radiation exhibiting unparalleled flexibility for both sets of applications.

Important classes of entangled two-photon states include those exhibiting positive frequency correlations 2 . 3], required for certain metrological applications, and those characterized by vanishing correlations in all degrees of freedom, crucial for applications that require the concatenation of several sources, such as linear optical quantum computation [4]. In most PDC sources to date, however, the photon pair directions and frequencies are strongly correlated, leading to space-time entanglement in the joint quantum state. This means that when one photon is detected, the other is projected into a mixed state, which will not interfere with other photons [5, 6]. Experimental proposals for generating factorable states have been recently reported $6,[7,[8]$; these tend to rely on favorable material properties occurring at specific wavelengths. A more general method is to engineer the photonic state by coherent manipulation of the probability amplitudes for generating photons of particular frequency and/or direction.

The synthesis of two-photon states characterized by a joint amplitude of arbitrary shape in two-dimensional signal and idler frequency space $\omega_{s}-\omega_{i}$ requires the use of a broadband pump. A narrow bandwidth pump centered at $\omega_{p}$ with width $\epsilon$ gives rise to photon pairs whose frequencies are nearly perfectly anti-correlated such that $\left|\omega_{s}+\omega_{i}-\omega_{p}\right| \leq \epsilon / 2$. Thus, for example, broadband states exhibiting vanishing or positive spectral correlations cannot be constructed within such a one-dimensional subspace; use of a broadband pump frees us from this restriction. Work by Klyshko and others 9, 10] has explored the potential for PDC state engineering of compensating dispersive effects in nonlinear crystals by appropriately chosen linear media. Here we build upon this pioneering work, presenting both theoretically and experimentally a technique which relies on a "superlattice" of nonlinear and anisotropic linear segments, pumped by an ultrashort pulse, which permits the generation of photon pairs with an arbitrary degree of entanglement via appropriate group-delay engineering. When used in waveguided geometries, where spectral and spatial degrees of freedom are decoupled 11] making large collection apertures possible, the technique presented here can lead to bright photon pair sources with customizable modal character.

The key to this method is an extension of the notion of group velocity matching that was introduced for the generation of spectrally decorrelated two-photon states 17, 12, to the idea of manipulating overall group delay mismatches between the various fields in structured materials. Let us note that a pulsed pump provides a timing reference for the PDC photons, not present in the CW case. Indeed, the ability to arbitrarily select the orientation in $\omega_{s}-\omega_{i}$ space of the joint spectral amplitude (thus permitting an arbitrary degree of entanglement) requires, in the pulsed case, control over two group delays (pump-signal and pump-idler) compared to a single group delay (signal-idler) in the $\mathrm{CW}$ case. Therefore, in general, two-dimensional group delay mismatch control is required, which can be achieved by interspersing nonlinear superlattice segments with linear segments exhibiting 
an appropriate birefringence.

To be concrete, the two-photon state produced by spontaneous PDC for fixed propagation directions [13] is:

$$
|\Psi\rangle=A \int_{0}^{\infty} \int_{0}^{\infty} d \omega_{s} d \omega_{i} f\left(\omega_{s}, \omega_{i}\right) a_{s}^{\dagger}\left(\omega_{s}\right) a_{i}^{\dagger}\left(\omega_{i}\right)|0\rangle_{s}|0\rangle_{i}
$$

where $A$ is a normalization constant, $a_{\mu}^{\dagger}\left(\omega_{\mu}\right)$ (with $\mu=s, i)$ is the creation operator for the two PDC modes and $f\left(\omega_{s}, \omega_{i}\right)$ is the joint spectral amplitude (JSA) given by the product of the pump envelope function (PEF) $\alpha\left(\omega_{s}+\omega_{i}\right)$ and the phasematching function (PMF) $\phi\left(\omega_{s}, \omega_{i}\right)$ [13]. The normalized joint spectral intensity (JSI) $\left|f\left(\omega_{s}, \omega_{i}\right)\right|^{2}$ represents a probability density function for PDC emission at specific frequencies $\omega_{s}$ and $\omega_{i}$.

Our source design is based on a periodic assembly of standard $\chi^{(2)}$ crystals and linear birefringent spacers. Consider the PDC source shown in Fig. 1] consisting of $N$ identical $\chi^{(2)}$ crystals of length $L$ (cut and oriented for degenerate collinear type-II PDC centered at $\omega_{0}$ ) and $N-1$ identical linear $\chi^{(1)}$ spacer crystals of length $h$. The PMF for such a superlattice is given by $[\underline{6},[9]$ :

$$
\phi_{N}\left(\nu_{s}, \nu_{i}\right) \propto \frac{\sin \left[N \Phi\left(\nu_{s}, \nu_{i}\right) / 2\right]}{\sin \left[\Phi\left(\nu_{s}, \nu_{i}\right) / 2\right]} \operatorname{sinc}\left[L \Delta k\left(\nu_{s}, \nu_{i}\right) / 2\right]
$$

where $\nu_{s, i}=\omega_{s, i}-\omega_{0}$,

$$
\Phi\left(\nu_{s}, \nu_{i}\right)=L \Delta k\left(\nu_{s}, \nu_{i}\right)+h \Delta \kappa\left(\nu_{s}, \nu_{i}\right)
$$

and where $\Delta k\left(\nu_{s}, \nu_{i}\right)$ and $\Delta \kappa\left(\nu_{s}, \nu_{i}\right)$ are the phase mismatches in the crystal and spacer segments:

$$
\begin{aligned}
& \Delta k\left(\nu_{s}, \nu_{i}\right)=k_{p}\left(\nu_{s}+\nu_{i}\right)-k_{s}\left(\nu_{s}\right)-k_{i}\left(\nu_{i}\right) \\
& \Delta \kappa\left(\nu_{s}, \nu_{i}\right)=\kappa_{p}\left(\nu_{s}+\nu_{i}\right)-\kappa_{s}\left(\nu_{s}\right)-\kappa_{i}\left(\nu_{i}\right) .
\end{aligned}
$$

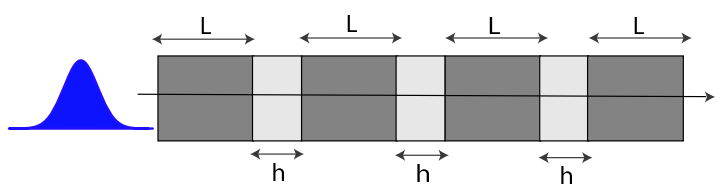

FIG. 1: Nonlinear crystal superlattice, pumped by an ultrashort pulse, yielding an arbitrary degree of entanglement.

Hence, the total PMF is composed of the product of two separate functions: one due to each individual crystal segment and one due to the superlattice. A change of variables to $\nu_{ \pm}=2^{-\frac{1}{2}}\left(\nu_{s} \pm \nu_{i}\right)$ and a Taylor expansion of the crystal and spacer phasemismatches yields:

$$
\Phi\left(\nu_{+}, \nu_{-}\right)=h \Delta \kappa^{(0)}+L \Delta k^{(0)}+\tau_{+} \nu_{+}+\tau_{-} \nu_{-},
$$

where $\Delta k^{(0)}$ and $\Delta \kappa^{(0)}$ are evaluated at $\omega_{0}$ and:

$$
\begin{aligned}
& \tau_{+}=2^{-\frac{1}{2}}\left[L\left(k_{s}^{\prime}+k_{i}^{\prime}-2 k_{p}^{\prime}\right)+h\left(\kappa_{s}^{\prime}+\kappa_{i}^{\prime}-2 \kappa_{p}^{\prime}\right)\right] \\
& \tau_{-}=2^{-\frac{1}{2}}\left[L\left(k_{s}^{\prime}-k_{i}^{\prime}\right)+h\left(\kappa_{s}^{\prime}-\kappa_{i}^{\prime}\right)\right] .
\end{aligned}
$$

Here $\kappa_{\mu}^{\prime}$ and $k_{\mu}^{\prime}(\mu=p, s, i)$ denote the reciprocal group velocities (RGV) in the spacer and nonlinear material respectively. We assume that $\Delta k^{(0)}=0$, thus ensuring phasematching in the crystal segments. The resulting superlattice JSA can be engineered by noting that whereas the orientation of the single crystal contribution on the $\nu_{s}-\nu_{i}$ plane is fixed, that of the crystal sequence can be adjusted by varying the relative thicknesses of the crystal and spacer segments $[$. Our approach is to let the single crystal PMF be much broader than the spectral structure imposed by the superlattice, so that the latter determines the resulting two-photon state properties. Under these circumstances and modelling the PEF $\alpha\left(\omega_{s}+\omega_{i}\right)$ as a Gaussian function with width $\sigma$ we obtain:

$$
f\left(\nu_{+}, \nu_{-}\right) \propto \frac{\sin \left[N \Phi\left(\nu_{+}, \nu_{-}\right) / 2\right]}{\sin \left[\Phi\left(\nu_{+}, \nu_{-}\right) / 2\right]} \exp \left[-2 \nu_{+}^{2} / \sigma^{2}\right]
$$

with $\Phi\left(\nu_{+}, \nu_{-}\right)$given by Eq. 5 Let us note that rotating by $180^{\circ}$ subsequent crystal segments, thus flipping the incidence angle relative to the optic axis, it is possible to limit the maximum transverse walkoff to its single cyrstal value (spacer segments exhibit no walkoff for propagation parallel or orthogonal to the optic axis). Alternatively, a waveguided geometry evidently suppresses spatial walkoff. Simultaneous temporal and spatial matching between pump, signal and idler is indeed a powerful tool for the engineering of two-photon states.

The JSA given by Eq. 7 may be adapted to produce various photonic states. This may be illustrated by an example. States with symmetric JSAs [i.e. $f\left(\omega_{s}, \omega_{i}\right)=$ $f\left(\omega_{i}, \omega_{s}\right)$ ] exhibit perfect visibility in two-photon HongOu-Mandel (HOM) interference, an essential ingredient in many optical QIP protocols. Eqns. 7 and [5] reveal that by imposing the condition $\tau_{-}=0, \nu_{-}$dependence is suppressed; the former leads to a symmetric JSA, in particular one exhibiting frequency anticorrelation (which may be very tight for a long superlattice) which satisfies the HOM symmetry condition despite the fact that, individually, each type-II crystal does not. In our approach, the spectral(temporal) distinguishing information from individual crystal segments is eliminated by compensating the crystal signal-idler temporal walkoff $L\left(k_{s}^{\prime}-k_{i}^{\prime}\right)$ by that in the linear birefringent spacers $h\left(\kappa_{s}^{\prime}-\kappa_{i}^{\prime}\right)$, which ensures the suppression of the overall signal-idler temporal walkoff term $\tau_{-}$. Thus, crystal and spacer materials must be chosen with opposite signed signal-idler walkoffs while segment thicknesses must be selected appropriately.

A JSA of this type can be engineered using BBO crystal segments cut for degenerate collinear type-II PDC at $800 \mathrm{~nm}$ (with $42.4^{\circ}$ cut angle) and quartz spacers (BBO and quartz fast axes oriented orthogonally). For BBO segments $k_{e}^{\prime}-k_{o}^{\prime}=-194 \mathrm{fs} / \mathrm{mm}$, while for quartz spacers $\kappa_{e}^{\prime}-\kappa_{o}^{\prime}=31 \mathrm{fs} / \mathrm{mm}$; thus, the spacer to crystal thickness ratio must be $h / L \approx 6.1$ in order that $\tau_{-}=0$ (the plots in Fig. 2] assume $L=250 \mu \mathrm{m}$ and $h=1.53 \mathrm{~mm}$ ). Fig. 2(A) shows the single crystal PMF, which does not satisfy the HOM symmetry condition 14]. Fig. 2(B) shows the superlattice PMF contribution (left-hand fac- 

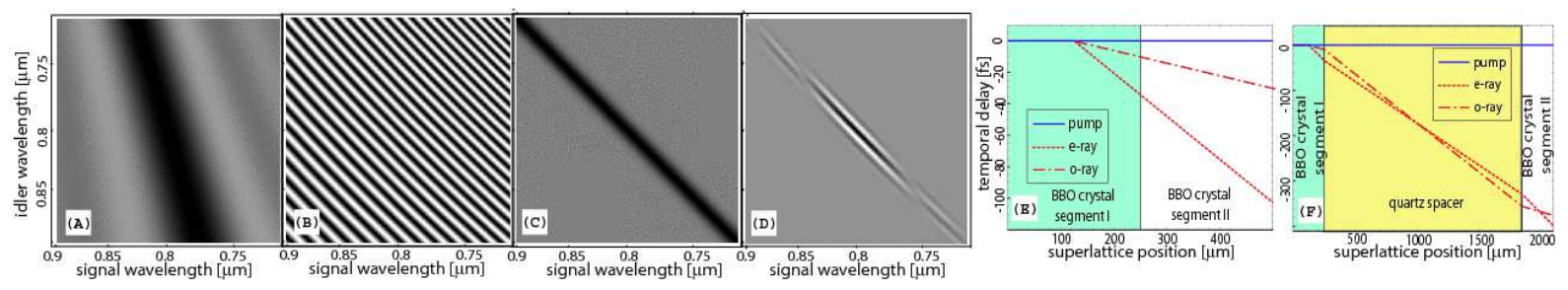

FIG. 2: Density plots of: (A) single crystal segment PMF exhibiting lack of symmetry, (B) BBO-quartz-BBO superlattice contribution to the PMF, (C) pump envelope function, (D) JSI symmetrized by the superlattice. (E)Time (with respect to pump pulse) at which signal/idler generated halfway through the first crystal traverse each point of two crystal segments. (F)Same as before for a BBO-quartz-BBO superlattice; signal-idler walkoff is limited to its single crystal value.

tor in Eq. 21). Fig. 2(C) shows the PEF (centered at 400nm with $1.95 \mathrm{~nm}$ bandwidth). Fig. 2(D) shows the resulting crystal-spacer-crystal superlattice JSI exhibiting the desired symmetry; the single crystal spectral bandwidth is sufficiently large that the superlattice determines the photon pair properties.

The group delay engineering above may be understood by considering how the quartz spacer limits the signalidler temporal walkoff. Fig. 2(E) shows for each point along a single $500 \mu \mathrm{m}$ thick crystal (considered as two $250 \mu \mathrm{m}$ crystals in sequence) the time, with respect to the pump pulse, at which each of the signal and idler wavepackets traverses each point of the crystal, assuming a photon pair created halfway through the first crystal. Fig. 2(F) shows the effect of the quartz spacer: it limits the maximum signal-idler walkoff to that observed in a single $250 \mu \mathrm{m}$ crystal (it increases, however, the pumpPDC walkoff, which does not impact the HOM symmetry condition). Thus, even though signal-idler temporal walkoff cannot be entirely suppressed, it can be limited in a superlattice to an arbitrarily small value.

In order to illustrate the power of this concept, we implemented the simple superlattice described above. The experimental configuration used [see Fig. 3] relies on a collinear type II HOM interferometer (HOMI). A train of ultrashort pulses centered at 405nm (2nm FWHM bandwidth, $82 \mathrm{MHz}$ repetition rate and $150 \mathrm{~mW}$ power), obtained by frequency doubling a mode-locked Ti:Sapphire beam, was weakly focused onto a collinear type II PDC source. The latter is based on $250 \mu \mathrm{m}$ length BBO crystal segments, and could be configured as single-crystal, double-crystal or as a crystal-quartz-crystal superlattice. A polarizing beamsplitter (PBS1) established a Michelson interferometer, each polarization in one arm. Quarter wave plates (QWP1/QWP2) reverse the polarization, so that both photons exit PBS1 from its second port. One of the arm lengths was adjustable via a motorized translation stage (TS). The PDC exiting the interferometer was coupled into a single mode fiber (SMF) which ensured a high degree of signal-idler spatial modematching, prior to which a dichroic beamsplitter (blue reflecting/red transmitting) followed by a red-colored glass filter, separated the PDC light from the blue pump. A half-wave plate (HWP) oriented the orthogonal signal/idler modes at $45^{\circ}$ with respect to the axes of a sec-

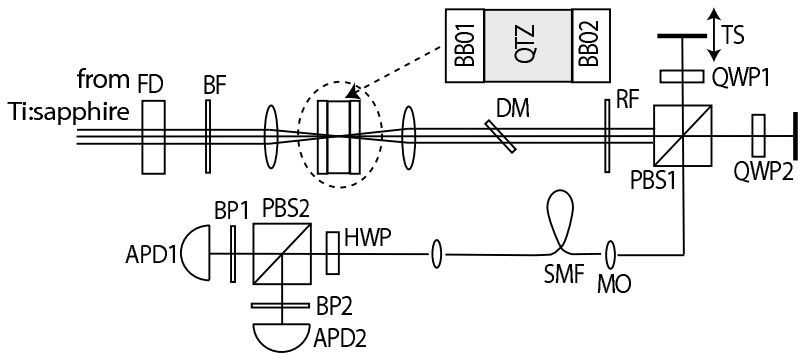

FIG. 3: BBO1/BBO2: 250 $\mu \mathrm{m}$ BBO crystals, QTZ: $1.53 \mathrm{~mm}$ quartz spacer, PBS1/PBS2: polarizing beam splitters, QWP1/QWP2: quarter waveplates, TS: translatable mirror, MO: $16 \times$ microscope objetive, SMF: single mode fiber, HWP: half wave plate, BP1/BP2: bandpass filters of 40nm width, APD1/APD2: avalanche photo diodes.

ond polarizing beam splitter (PBS2) which formed the basis for a collinear HOMI. Each output mode from the PBS was focused on the active area of a silicon photoncounting module; wide-bandpass filters (wider than the PDC bandwidth) were used for background suppression. Data was taken by monitoring coincidence counts as a function of the translation stage (TS) position.

The data in Fig. प 4 shows coincidence count rate data, normalized to a unit background, as a function of the signal-idler relative delay. Solid curves represent theoretical curves obtained from a calculation similar to that in Ref. [13]. Fig. [4(A) shows the interference curve resulting from a single crystal segment. Fig. 4(B) shows a broadened dip with reduced visibility, due to the increased signal-idler temporal walkoff [quantified by $\tau_{-}$, Eq. 6, resulting from two crystal segments in sequence. Fig. 4(C) shows the result of placing a $1.6 \mathrm{~mm}$ length quartz spacer between the crystals [see Fig. 3]. Note that the visibility and dip width are restored to their single crystal values, thus demonstrating the effect of limiting the maximum walkoff to that experienced by a single crystal. Fig. 4(D) shows the effect of rotating the quartz spacer by $90^{\circ}$, thus reversing the extraordinary and ordinary character in the spacer. In this latter case, the superlattice contribution is rotated away from the diagonal axis on the $\omega_{s}-\omega_{i}$ plane, thus destroying fulfilment of the HOM symmetry condition; the interference curve 


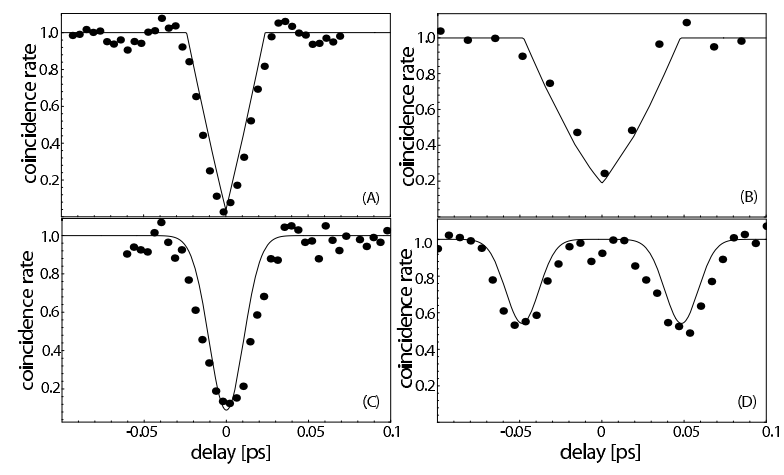

FIG. 4: HOM interference curves, normalized to a unit background to suppress source brightness variation resulting from re-alignment for each experiment. (A) single crystal segment. (B) Two crystal segments in sequence, exhibiting reduced visibility and broadening. (C) BBO-quartz-BBO superlattice exhibiting restoration of visibility. (D) $\mathrm{BBO}$-quartz-BBO with quartz rotated by $90^{\circ}$; visibility no longer restored.

now contains two dips, each associated with one of the crystals. Let us note that our conclusions depend on an analysis of HOM interference features and are independent of source brightness; however, a future refinement is the careful selection of the spacer thickness in order to rule out thickness-dependent count rate oscillations [17].

So far we have considered the effect of signal-idler temporal walkoff. In general the pump experiences a different group velocity from the signal and idler photons, so that there is a corresponding temporal walkoff between the pump and the PDC photon pairs. It is evident that the condition $\tau_{+}=0$ (see Eq. 6) closely resembles the group velocity matching (GVM) condition [7, 12] by which the pump RGV is matched to the mean RGV of the signal and idler wavepackets. In a superlattice, however, it is the total group delay mismatch which must vanish. This generalized condition can lead to spectrally factorable states, or to states exhibiting positive spectral correlations. For example, $\tau_{+}=0$ can be satisfied us- ing type-II BBO crystal segments and calcite spacers $[$ ] Note that the GVM condition fulfilled by the superlattice depends crucially on $L$ and $h$. The thickness error tolerance becomes more restrictive for longer assemblies (i.e. for higher $N$ ); for example for a superlattice of $10 \mathrm{BBO}$ crystals and 9 calcite spacers designed to yield a positive correlation state, the tolerance is about $2 \%$. In addition, the technique presented here can be adapted to produce states exhibiting a very large Schmidt number (and thus a high degree of space-time entanglement) 15] which constitute extreme examples of non-classical correlations 16.

In conclusion, we have shown that a periodic array of nonlinear crystals and linear birefringent spacers, pumped by an ultrashort pump, can result in PDC photon pairs exhibiting an engineered JSA function. We have illustrated this concept by demonstrating a source capable of high-visibility HOM interference, in a typeII pulsed pump configuration, using using materials that do not generate such states naturally. Our scheme has wider applicability; it can generate a broad class of two photon states including those with factorable and positively correlated JSAs, as well as those with a very large Schmidt number. It is conceivable that appropriate superlattices could be implemented in monolithic nonlinear optical structures where locally-modified dispersion characteristics are obtained by ion-exchange, doping or other mechanisms. This would open up a powerful new source engineering technique, akin to quasi phasematching but in the group velocity, rather than phase velocity, domain.

\section{Acknowledgments}

We acknowledge useful conversations with M. Fejer and M.G. Raymer. This work was supported by ARDA grant P-43513-PH-QCO-02107-1. AU acknowledges support from the Center for Quantum Information, funded by ARO administered MURI grant DAAG-19-99-1-0125.
[1] P. Michler et al., Science 290, 2282 (2000); Z. Yuan et al. Science 295, 102 (2002); C. Santori et al. , Nature 419, 594 (2002); C. Kurtsiefer et al., Phys. Rev. Lett. 85, 290 (2000); A. Kuhn et al., Phys. Rev. Lett. 89, 067901 (2002); B. Lounis et al., Nature 407, 491 (2000).

[2] V. Giovannetti et al., Nature, 412, 417 (2001); V. Giovannetti et al., Phys. Rev. Lett, 88, 183602 (2002).

[3] R. Erdmann et al., Phys. Rev. A, 62, 053810 (2000)

[4] E. Knill et al., Nature 409, 46 (2001); T.C. Ralph et al., Phys. Rev. A 65, 012314 (2001); T.B. Pittman et al., Phys. Rev. A 68, 032316 (2003).

[5] L. Zhang et al. in Continuous Variables in Quantum Optics,eds G. Leuchs and N. Cerf (World Scientific, Singapore, 2005).

[6] A.B. U'Ren et al., Las. Phys. 15146 (2005); A.B. U'Ren et al., J. Mod. Opt. 522197 (2005)
[7] W.P. Grice et al., Phys. Rev. A 64, 063815 (2001).

[8] A.B. U'Ren et al., Quant. Inf. and Comp. 3, 480 (2003); Z.D. Walton et al., Phys. Rev. A 70, 052317 (2004); O. Kuzucu et al., Phys. Rev. Lett. 94, 083601 (2005); J.P. Torres et al., Opt. Lett. 30, 314 (2005); M.G. Raymer et al., Phys. Rev. A 72, 023825 (2005).

[9] D.N. Klyshko JETP 77, 222 (1993).

[10] Di Giuseppe et al., Phys. Rev. A 66, 013801. (2002)

[11] A.B. U'Ren et al., Phys. Rev. Lett. 93, 093601 (2004).

[12] T.E. Keller et al., Phys. Rev. A 56, 1534 (1997).

[13] W.P. Grice et al., Phys. Rev. A 56, 1627 (1997).

[14] D. Branning et al., Phys. Rev. Lett. 83, 955 (1999).

[15] L. Zhang et al. accepted J. Mod. Opt.

[16] C.K. Law et al. Phys. Rev. Lett. 92, 127903 (2004).

[17] T.J. Herzog et al. Phys. Rev. Lett. 72, 629 (1994) 